(C) 1983. The Genetical Society of Great Britain

\title{
SELF-INCOMPATIBILITY IN LOLIUM SPECIES
}

\author{
2. LOLIUM PERENNE L. \\ J. M. MCCRAW AND W. SPOOR \\ Edinburgh School of Agriculture, West Mains Road, Edinburgh, EH9 3JG, U.K.
}

Received 23.viii.82

\section{SUMMARY}

An investigation of the genetic control of self-incompatibility in an $F_{1}$ family of $L$. perenne $\mathrm{L}$. using the pollen-stigma reaction indicated the involvement of more than two multiallelic loci. Pollen control was found to be gametophytically determined. Results indicated that the genetic control of self-incompatibility in $L$. perenne is similar to that in $L$. rigidum and $L$, multiflorum. It is suggested that not all alleles in pollen and pistil have to be matched to produce an incompatible reaction in Lolium species, in contrast to the system found in other self-incompatible grasses.

\section{INTRODUCTION}

Early investigations into the genetic control of self-incompatibility in the Gramineae revealed that the system was controlled at two multiallelic loci, with gametophytic determination of pollen behaviour. An incompatible reaction occurred only when both alleles present in the pollen were matched in the style (Lundqvist, 1954, 1955, 1956, 1961, 1962, 1965; Hayman, 1956). In the early 1970 s two more genera within the Gramineae were investigated. In one genus, Briza, the first species studied, $B$. media, was shown to have a two locus system similar in all respects to those described by Lundqvist and Hayman (loc. cit.) (Murray, 1974). However, examination of $B$. spicata (Murray, 1979) revealed results which could not be explained by the two multiallelic loci model. In addition, results from Lolium rigidum (McCraw and Spoor, 1983) and L. multiflorum (Hay, 1978; McCraw and Spoor, 1983) indicated a three locus control of the self-incompatibility system.

The genetic control of the self-incompatibility system of $L$. perenne has been variously postulated to be controlled by one or two loci (Hayward and Wright, 1971), two loci (Weimarck, 1968; Cornish, Hayward and Lawrence, 1979) or more than two loci (Spoor, 1976). However, Weimarck's (1968) data can be interpreted in terms of a one locus gametophytic incompatibility system. Hayward and Wright (1971), using a staining technique which made it difficult to distinguish between compatible and inviable pollen, found that it was not possible to interpret their results in terms of a one or two locus system. Cornish et al. (1979) and Spoor (1976) both used callose fluorescence to detect pollen tube growth through the stigma, and while Spoor (1976) classified results as compatible or incompatible only, Cornish et al. (1979) divided compatible pollinations into three types. Subdivision of compatible pollinations could be fraught with uncertainty since it has been demonstrated that less than the expected proportion of compatible pollen grains are frequently observed in crosses between plants of known genotype (Lundqvist, 1961; Hayman, 1956). 
Furthermore, by subdividing compatible pollinations into three classes based on a visual and not a numerical assessment, Cornish et al. (1979) may have overlooked any discrepancies.

This paper will deal with the results of a diallel cross carried out in one $F_{1}$ family of $L$.perenne and is the last study in this series of self-compatibility in Lolium species.

\section{MATERIALS AND METHODS}

The $F_{1}$ family was produced by controlled pollinations between two self-incompatible plants from different experimental lines of $L$. perenne $L$. supplied by the Welsh Plant Breeding Station. The $F_{1}$ family (designated family 6) contained twenty-three plants and was grown to flowering in a heated glasshouse in a 16-hour day.

The techniques of pollination, staining and observation were as previously described (McCraw and Spoor, 1983).

\section{Results}

The results of the diallel cross carried out for $F_{1}$ family 6 are given in fig. 1. Gametophytic determination of pollen behaviour was indicated by the observation of a non-uniform pollen reaction on the stigma in compatible crosses. However, it was not possible to classify compatible pollinations into distinct classes. More than half (11 out of 23 ) of the plants were self-compatible when pollinated in vitro. When these plants were allowed to self-pollinate in vivo, two seeds were produced by plant 5 while the other ten plants did not set any seed.

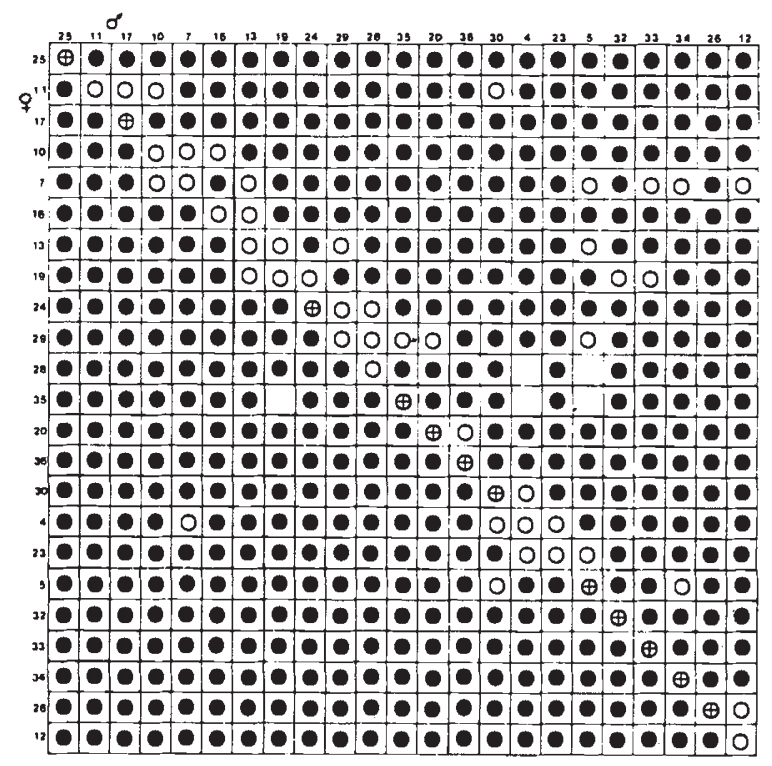

- Compatible 0 incompantible $\quad \oplus$ Sell.compatible

FIG. 1. Family 6: results of crosses between twenty-three plants produced by a controlled cross between two diploid $L$. perenne plants from different experimental lines. 
All but five of the possible crosses were carried out and the results show that four pairs of plants were reciprocally incompatible $(23 / 4,4 / 30$, $10 / 7,13 / 19$; fig. 1). In addition, 27 pairs of one way compatible reactions were observed, the remainder being reciprocally compatible.

\section{Discussion}

The results presented for the $F_{1}$ family of $L$. perenne are very similar to those already given for $L$. rigidum and $L$. multiflorum (McCraw and Spoor, 1983). The self-incompatibility system in the Lolium species investigated appears to be controlled by at least three multiallelic loci with gametophytic determination of pollen behaviour. Reciprocal incompatibility was observed between plants which had different self-incompatibility genotypes and it was therefore suggested that not all the alleles in the pollen and pistil have to be matched to cause an incompatible reaction (McCraw and Spoor, 1983). One way compatibility between pairs of plants may be explained if the parent plants used to produce the progeny examined had self-incompatibility alleles in common. The occurrence of common $\boldsymbol{S}$-alleles has previously been observed in Lolium and Ranunculus over considerable geographical distances (Cornish et al., 1979; Østerbye, 1977).

The results obtained in this study on the genetic control of self-incompatibility in $L$. perenne are in broad agreement with two earlier investigations. Hayward and Wright (1971) and Spoor (1976) both observed plants which were reciprocally incompatible and yet had different mating types, and examples of one way compatibility between pairs of plants. While Spoor (1976) observed more than sixteen different mating types and postulated a three locus system, Hayward and Wright (1971) found their results could not be explained by the two locus system postulated by Lundqvist (1954, 1955, 1956, 1961).

Cornish et al. (1979) also studied the genetic control of self-incompatibility in $L$. perenne but expected to find a two locus system. This expectation was based on the assumption that the genetic basis of self-incompatibility is usually similar in closely related groups. Nevertheless, exceptions to this general principle are known in the Solanaceae (one and two locus gametophytic self-incompatibility systems: Pandey, 1962; Abdalla and Hermsen, 1971), Boraginaceae (polygenic and heteromorphic self-incompatibility systems: Crowe, 1971; Varopoulos, 1979) and Caryophyllaceae (gametophytic and sporophytic self-incompatibility systems: Lundqvist, 1979). In addition, Lundqvist, Østerbye, Larsen and Linde-Laursen (1973) suggested that inbreeding of experimental material, especially grasses, may lead to homozygosity and hence the underestimation of the number of $S$-loci.

Despite the possibility of non-uniformity in the genetic control of the self-incompatibility system in the Gramineae, Cornish et al. (1979) used a technique of visually assessing the number of compatible pollen grains and recognised only four classes $(0,50,75$ or 100 per cent compatible). However, it had previously been discovered, by counting, that the proportion of compatible pollen grains observed was frequently less than that expected in crosses between plants of known genotypes (Lundqvist, 1961; Hayman, 1956). In addition, if the gametophytic self-incompatibility system was controlled by three loci and all alleles had to be matched in pollen 
and pistil to produce an incompatible reaction, the percentage compatible pollen could be $0,50,75,87.5$ or 100 per cent (McCraw, 1981). The percentage compatible pollen varies according to the number of $S$-alleles common to both plants and the distribution of the common alleles within the $S$-loci. Alternatively, in a three locus system where only two alleles (one $S$ and one $Z$ from $S^{\prime} S^{\prime \prime} Z$ ) have to be matched to produce an incompatible reaction ( $\varnothing$ sterbye et $a l ., 1980$ ), percentage compatible pollen could be $0,25,50,62.5,75$ or 100 per cent, depending on the distribution of and the number of common alleles among the $S$-loci of the two plants (McCraw, 1981). Weimarck (1968) studied self-incompatibility in a number of Graminaceous species and divided compatible pollinations into five classes: $0,25,50,75$ and 100 per cent compatible. Significantly, a proportion of 25 per cent compatible pollen cannot be explained by the two locus systems proposed by Lundqvist $(1954,1955,1956,1961)$ and Hayman (1956) but it can be explained by the system proposed by Østerbye et al. (1980). Cornish et al. (1979) admit to misclassifying 9 per cent of pollinations observed, confusion arising mainly between 50 and 75 per cent compatible classes. Therefore, if either of the two alternative systems discussed here controlled the incompatibility system in $L$. perenne, it would be unlikely to be detected by visual assessment of pollen behaviour.

In conclusion, it appears that the genetic basis of self-incompatibility is similar in the three outbreeding Lolium species studied, namely three multiallelic loci with gametophytic determination of pollen behaviour and, in addition, the possibility that not all alleles in pollen and pistil have to be matched to produce an incompatible reaction. Although a two locus system was demonstrated for Briza media (Murray, 1974), a three locus system may be present in $B$. spicata (Murray, 1979). Therefore the Gramineae family appears to be another exception to the general principle that the genetic basis of self-incompatibility is the same within closely related groups.

Acknowledgements.-Grants from the 1969 Studentship in Agriculture, University of Edinburgh and Studley College Trust are gratefully acknowledged.

\section{REFERENCES}

ABDAlla, M. M. F. AND HERMSEN, J. G. TH. 1971. A two-loci system of gametophytic incompatibility in Solanum phureja and S. stenotomum. Euphytica, 20, 345-350.

CORNISH, M. A., HAYWARD, M. D. AND LAWRENCE, M. J. 1979. Self-incompatibility in ryegrass. 1. Genetic control in diploid Lolium perenne L. Heredity, 43, 95-106.

CROWE, L. K. 1971. The polygenic control of outbreeding in Borago officinalis. Heredity, 27, 111-118.

HAY, J. M. 1978. Incompatibility in Lolium multiflorum. Incompat. Newsletter, 10, 134-136. HAYMAN, D. C. 1956. The genetical control of incompatibility in Phalaris coerulescens, Desf. Aust. J. Biol. Sci., 9, 321-331.

HAYWARD, M. D. AND WRIGHT, A. J. 1971. The genetic control of incompatibility in Lolium perenne L. Genetica, 42, 414-421.

LUNDQVIST, A. 1954. Studies on self-sterility in rye, Secale cereale L. Hereditas, 40, 278-294. LUNDQVIST, A. 1955. Genetics of self-incompatibility in Festuca pratensis Huds. Hereditas, $41,518-520$.

LUNDQVIST, A. 1956. Self-incompatibility in rye. 1. Genetic control in the diploid. Hereditas, 42, 293-348.

LUNDQVIST, A. 1961. Self-incompatibility in Festuca pratensis Huds. Hereditas, 47, 542-562. 
LUNDQViST, A. 1962. Self-incompatibility in diploid Hordeum bulbosum L. Hereditas, 48, $138-152$.

LUNDQVIST, A. 1965. Self-incompatibility in Dactylis aschersoniana Graebn. Hereditas, 54, 70-87.

LUNDQVIST, A. 1979. One locus sporophytic self-incompatibility in the carnation family, Caryophyllaceae. Hereditas, 91, 307.

LUNDQViST, A., ØSTeRbye, U., LARSEN, K. AND linde-laursen, I. 1973. Complex self-incompatibility systems in Ranunculus acris L. and Beta vulgaris L. Hereditas, 74, 161-168.

McCRAW, J. M. 1981. Self-incompatibility in Lolium species. Ph.D. thesis, Edinburgh.

McCRAW, J. M. AND SPOOR, W. 1983. Self-incompatibility in Lolium species. 1. Lolium rigidum Gaud. and $L$. multiflorum L. Heredity 50, 1983 21-27.

MURRAY, B. G. 1974. Breeding systems and floral biology in the genus Briza. Heredity, 33, 285-292.

MURRAY, B. G. 1979. The genetics of self-incompatibility in Briza spicata. Incompat. Newsletter, $11,42-45$.

ØSTERBYE, U. 1977. Self-incompatibility in Ranunculus acris L. II. Four S-loci in a German population. Hereditas, 87, 173-178.

ØSTERBYE, U., LARSEN, K. AND LUNDQVIST, A. 1980. Comments on self-incompatibility in the Gramineae. Incompat. Newsletter, 12, 45-49.

PANDEY, K. K. 1962. Genetics of incompatibility behaviour in the Mexican Solanum species S. pinnatisectum $Z$. Vererb. Lehre, 93, 378-388.

SPOOR, w. 1976. Self-incompatibility in Lolium perenne L. Heredity, 37, 417-421.

VAROPOULOS, A. 1979. Breeding systems in Myosotis scorpioides L. (Boraginaceae). 1. Self-incompatibility. Heredity, 42, 149-157.

WEIMARCK, A. 1968. Self-incompatibility in the Gramineae. Hereditas, 60, 157-166. 